\title{
Some a priori estimates for a class of operators in the Heisenberg group
}

\author{
Fausto Ferrari
}

Received: 22 May 2012 / Accepted: 10 December 2012 / Published online: 23 December 2012

(C) Fondazione Annali di Matematica Pura ed Applicata and Springer-Verlag Berlin Heidelberg 2012

\begin{abstract}
In this note, we provide some a priori estimates for a class of operators that stem from the Laplacian in the Heisenberg group. We follow the idea contained in a proof given by Talenti, see (Ann Scuola Norm Sup Pisa Cl Sci (4) 3(4): 697-718, 1976), by adapting the classical notion of symmetrized rearrangement of a function to the framework of the Heisenberg group.
\end{abstract}

Keywords Heisenberg group - Maximum principle - Fully nonlinear operators · Rearrangements · Convex/concave operators

Mathematics Subject Classification $35 \mathrm{~J} 60 \cdot 35 \mathrm{~J} 70$

\section{Contents}

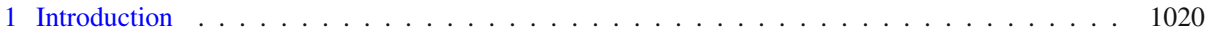

2 Some preliminaries . . . . . . . . . . . . . . . . . . . . . . . . . . . . . 1024

2.1 Properties of the gauge distance . . . . . . . . . . . . . . . . . . . . . . . . . . . . . 1024

2.2 Rearrangements . . . . . . . . . . . . . . . . . . . . . . . . . . . . . . . . 1028

3 Proof of the main results . . . . . . . . . . . . . . . . . . . . . . . . . . . . . 1030

3.1 The real part of the Kohn-Laplace operator case . . . . . . . . . . . . . . . . . . . . . . 1030

3.2 The case of degenerate operators in divergence form . . . . . . . . . . . . . . . . . . 1033

4 Some applications to some nonlinear operators . . . . . . . . . . . . . . . . . . . . . . . 1036

4.1 Properties of degenerate nonlinear uniformly elliptic operators . . . . . . . . . . . . . . . 1037

4.2 Some partial results: concave/convex operators . . . . . . . . . . . . . . . . . . . . . 1037

References . . . . . . . . . . . . . . . . . . . . . . . . . . . 1040

F. Ferrari

Dipartimento di Matematica dell' Università di Bologna,

Piazza di Porta S. Donato, 5, Bologna 40126, Italy

e-mail: fausto.ferrari@unibo.it 


\section{Introduction}

In this note, we deal with the existence of a priori estimates for a class of operators in the Heisenberg group using the approach followed by Talenti in [23], for nondegenerate elliptic operators in divergence form, adapting the classical notion of symmetrized rearrangement.

To introduce the argument, we recall that $\Delta_{\mathbb{H}^{n}}$, the sub-Laplacian in the Heisenberg group, is a linear operator that can be written both in divergence and in nondivergence form. For example, in the simplest case, namely in $\mathbb{H}^{1}$, we get, if $x=(\xi, \eta, \sigma) \in \mathbb{R}^{3} \equiv \mathbb{H}^{1}$ :

$$
\begin{aligned}
\Delta_{\mathbb{H} 1} u(x) & =\operatorname{div}\left(\left[\begin{array}{lll}
1, & 0, & 2 \eta \\
0, & 1, & -2 \xi \\
2 \eta, & -2 \xi, & 4\left(\xi^{2}+\eta^{2}\right)
\end{array}\right] \nabla u(x)\right) \\
& =\operatorname{Tr}\left(\left[\begin{array}{lll}
1, & 0, & 2 \eta \\
0, & 1, & -2 \xi \\
2 \eta, & -2 \xi, & 4\left(\xi^{2}+\eta^{2}\right)
\end{array}\right] D^{2} u(x)\right),
\end{aligned}
$$

where $\nabla u(x)$ and $D^{2} u(x)$ denote the usual gradient and the Hessian matrix of $u$ in $x$, respectively.

Notice that in the previous formula, we did not use any intrinsic notation. Indeed, we could write the same operator as follows:

$$
\operatorname{div}_{\mathbb{H}^{1}}\left(\nabla_{\mathbb{H}^{1}} u\right)=\Delta_{\mathbb{H}^{1}} u,
$$

where $\Delta_{\mathbb{H}^{1}} u=X^{2} u+Y^{2} u$ is the real part of the Kohn-Laplace operator in the Heisenberg group and $X, Y$ are the vector fields that generate the Lie algebra in the Heisenberg group that we are going to introduce later. In particular, $\operatorname{div}_{\mathbb{H}^{1}}$ acts on $b_{1} X+b_{2} Y$ as follows: $\operatorname{div}_{\mathbb{H}^{1}}\left(b_{1} X+b_{2} Y\right)=X b_{1}+Y b_{2}$. Here, we just point out that in each point $x \in \mathbb{H}^{1}$, the smallest eigenvalue of the matrix

$$
\left[\begin{array}{ccc}
1, & 0, & 2 \eta \\
0, & 1, & -2 \xi \\
2 \eta, & -2 \xi, & 4\left(\xi^{2}+\eta^{2}\right)
\end{array}\right]
$$

is zero.

We introduce in short the $n$-th Heisenberg group $\mathbb{H}^{n}$, namely as $\mathbb{R}^{2 n+1}$ endowed with the following noncommutative law: for every $(\xi, \eta, \sigma),\left(\xi^{\prime}, \eta^{\prime}, \sigma^{\prime}\right) \in \mathbb{R}^{n} \times \mathbb{R}^{n} \times \mathbb{R}$

$$
(\xi, \eta, \sigma) \circ\left(\xi^{\prime}, \eta^{\prime}, \sigma^{\prime}\right)=\left(\xi+\xi^{\prime}, \eta+\eta^{\prime}, \sigma+\sigma^{\prime}+2\left(\xi^{\prime} \cdot \eta-\eta^{\prime} \cdot \xi\right)\right),
$$

where $\xi \cdot \eta$ denotes the usual inner product in $\mathbb{R}^{n}$.

A dilation group in this framework is also defined as follows. For every $(\xi, \eta, t) \in \mathbb{H}^{n}$ and for every $r>0$

$$
\delta_{r}(\xi, \eta, \sigma)=\left(r \xi, r \eta, r^{2} \sigma\right) .
$$

Hence, the real part of the Kohn-Laplace operator is

$$
\Delta_{\mathbb{H}^{n}}=\sum_{i=n}^{2 n}\left(X_{i}^{2}+Y_{i}^{2}\right),
$$

where for $i=1, \ldots, n X_{i}=\partial_{\xi_{i}}+2 \eta_{i} \partial_{\sigma}, Y_{i}=\partial_{\eta_{i}}-2 \xi_{i} \partial_{\sigma}$.

Note that, as in the $n=1$ case, the smallest eigenvalue of the matrix associated with the real part of the Kohn-Laplace operator is always zero in all of $\mathbb{H}^{n} \equiv \mathbb{R}^{2 n+1}$. Thus, this operator is degenerate at each point of $\mathbb{H}^{n}$. 
A comprehensive description of the Heisenberg group can be found in [7] and in [10]. Hereafter, we recall just its main properties. In particular, we stress that the vector fields $X_{i}, Y_{i}, i=1, \ldots, n$ do not commute. Indeed, for any $i=1, \ldots, n$

$$
\left[X_{i}, Y_{i}\right]=-4 \partial_{\sigma}
$$

and as a consequence, formally, the definition of second-order objects, like the Hessian matrix, is not straightforward.

However, since we are mainly interested in operators that can be described as trace operator, we would like to deal with symmetric matrices in order to get real eigenvalues. Thus, it is useful to introduce the symmetric horizontal Hessian matrix modeled on the Lie algebra of the Heisenberg group as follows:

$$
D_{\mathbb{H}^{n}}^{2 *} u=\frac{D_{\mathbb{H}^{n}}^{2} u+\left(D_{\mathbb{H}^{n}}^{2} u\right)^{T}}{2},
$$

where

$$
D_{\mathbb{H}^{n}}^{2} u=\left[\begin{array}{c}
X_{1} \nabla_{\mathbb{H}^{n}} u \\
X_{2} \nabla_{\mathbb{H}^{n}} u \\
\vdots \\
X_{n} \nabla_{\mathbb{H}^{n}} u \\
Y_{1} \nabla_{\mathbb{H}^{n}} u \\
Y_{2} \nabla_{\mathbb{H}^{n}} u \\
\vdots \\
Y_{n} \nabla_{\mathbb{H}^{n}} u
\end{array}\right],
$$

and $\nabla_{\mathbb{H}^{n}} u=\left(X_{1} u, \ldots, X_{n} u, Y_{1} u, \ldots, Y_{n} u\right)$. Hence, let $A$ be a $2 n \times 2 n$ matrix. If we define

$$
\mathcal{L}_{\mathbb{H}^{n}, A} u=\operatorname{Tr}\left(A D_{\mathbb{H}^{n}}^{2} u\right),
$$

then, whenever $A=A^{T}$, we also get that

$$
\mathcal{L}_{\mathbb{H}^{n}, A} u=\operatorname{Tr}\left(A D_{\mathbb{H}^{n}}^{2 *} u\right)
$$

The intrinsic divergence of a smooth horizontal vector field $V=\sum_{i}^{2 n}\left(p_{i} X_{i}+q_{i} Y_{i}\right)$ in $\mathbb{H}^{n}$ is defined as

$$
\operatorname{div}_{\mathbb{H}^{n}} V=\sum_{i}^{2 n}\left(X_{i} p_{i}+Y_{i} q_{i}\right)
$$

In particular, the gradient $\nabla_{\mathbb{H}^{n}} u$ of a smooth function $u$ is

$$
\operatorname{div}_{\mathbb{H}^{n}}\left(\nabla_{\mathbb{H}^{n}} u\right)=\sum_{i=1}^{n}\left(X_{i}\left(X_{i} u\right)+Y_{i}\left(Y_{i} u\right)\right),
$$


so that $\operatorname{div}_{\mathbb{H}^{n}}\left(\nabla_{\mathbb{H}^{n}} u\right)=\Delta_{\mathbb{H}^{n}} u$. Hence, for a smooth $2 n \times 2 n$ matrix $A=\left(a_{i, j}\right)_{1 \leq i, j \leq 2 n}$, it is well defined the following operator in divergence form:

$$
\begin{aligned}
\operatorname{div}_{\mathbb{H}^{n}}\left(A(x) \nabla_{\mathbb{H}^{n}} u\right)= & \sum_{i=1}^{n}\left(X_{i}\left(\sum_{j=1}^{n} a_{i, j}(x) X_{j} u+a_{i, n+j}(x) Y_{j} u\right)\right. \\
& \left.+Y_{i}\left(\sum_{j=1}^{n} a_{n+i, j}(x) X_{j} u+a_{n+i, n+j}(x) Y_{j} u\right)\right) .
\end{aligned}
$$

We denote moreover with $Q=2 n+2$ the homogeneous dimension in the Heisenberg group $\mathbb{H}^{n}$, see [7], and with $d_{K}$ the so-called gauge distance, defined starting from the norm:

$$
d_{G}((\xi, \eta, \sigma))=\sqrt[4]{\left(|\xi|^{2}+|\eta|^{2}\right)^{2}+\sigma^{2}} .
$$

Indeed the gauge distance, namely the so-called Koranyi distance that must not be confused with the Carnot-Charathéodory distance and to whom is, however, equivalent, is defined as $d_{K}(x, y)=d_{G}\left(x^{-1} \circ y\right)$. Concerning the definition of Carnot-Charathéodory distance and its property, we refer to [7], see also [2-4] for some topics. We just remind that a inner product on the horizontal tangent space at each point $x \in \mathbb{H}^{n}$ can be introduced. More precisely, for every $V(x)=\sum_{i=1}^{n}\left(v_{i}(x) X_{i}(x)+v_{n+i}(x) Y_{i}(x)\right)$ and for every $W(x)=\sum_{i=1}^{n}\left(w_{i}(x) X_{i}(x)+\right.$ $\left.w_{n+i}(x) Y_{i}(x)\right)$, where $\left(v_{1}, \ldots, v_{2 n}\right): \mathbb{H}^{n} \rightarrow \mathbb{R}^{2 n}$, and $\left(w_{1}, \ldots, w_{2 n}\right): \mathbb{H}^{n} \rightarrow \mathbb{R}^{2 n}$, we define

$$
\langle V(x), W(x)\rangle_{\mathbb{H}^{n}}=\sum_{i=1}^{n}\left(v_{i}(x) w_{i}(x)+v_{n+i}(x) w_{n+i}(x)\right) .
$$

By the way, in order to avoid confusion, all the intrinsic objects in the $n$-Heisenberg framework will be labeled with the symbol $\mathbb{H}^{n}$.

Now we can introduce our main result.

Theorem 1.1 Let $\Omega \subset \mathbb{H}^{n}$ be an open bounded set and $f \in L^{Q}(\Omega)$. Let $A$ be a positive definite, symmetric $2 n \times 2 n$ matrix.

If $u$ is a solution of

$$
\operatorname{div}_{\mathbb{H}^{n}}\left(A(x) \nabla_{\mathbb{H}^{n}} u(x)\right)=f
$$

in $\Omega$, then

$$
\sup _{\Omega} u \leq \sup _{\partial \Omega} u+C(n, \lambda)|\Omega|^{\frac{1}{Q}}\|f\|_{L^{Q}(\Omega)},
$$

where $\lambda>0$ is the smallest eigenvalue of $A$ in $\Omega$ and $Q=2 n+2$.

Moreover, let $\lambda>0$ be fixed. There exists a positive constant $C=C(n, \lambda)$ such that if $u \in C^{2}(\Omega) \cap C(\bar{\Omega})$, then for every $2 n \times 2 n$ constant matrix $A, A \geq \lambda I, \lambda>0$,

$$
\sup _{\Omega} u \leq \sup _{\partial \Omega} u+C(n, \lambda)|\Omega|^{\frac{1}{Q}}\left\|\operatorname{Tr}\left(A D_{\mathbb{H}^{n}}^{2} u\right)\right\|_{L^{Q}(\Omega)}
$$

moreover, if $A$ is also symmetric, then

$$
\sup _{\Omega} u \leq \sup _{\partial \Omega} u+C(n, \lambda)|\Omega|^{\frac{1}{Q}}\left\|\operatorname{Tr}\left(A D_{\mathbb{H}^{n}}^{2 *} u\right)\right\|_{L^{Q}(\Omega)} .
$$


To prove Theorem 1.1, we use symmetrisation techniques. This result is, in a sense, equivalent to the one obtained by Gutierrez and Lanconelli for the $X$-Elliptic operators, see Theorem 3.1 in [19]. However, in our proof, we will use a different approach to treat the subject, and this is one of the main reasons to write this note. Indeed, in order to avoid misunderstanding, we point out that $X$-Elliptic operators define a wide class of operators in divergence form even containing the sub-Laplacian of the Heisenberg group. Nevertheless, we think that our method might be useful to improve the knowledge about the existence of a sharp maximum principle even for degenerate operators in nondivergence form.

Few words concerning the literature about this subject are in order. The perspective mainly applied to study this subject has been, until now, to understand what are in the Heisenberg framework the intrinsic geometric objects that might be used to mimic the Euclidean proof of the maximum principle for nondivergence operators. Indeed, in [20] see Theorem 1.3, using the notion of intrinsic convex function in the Heisenberg group, see also [12], it has been proved an inequality that the authors called 'Maximum principle'.

Roughly speaking, the authors state that the square of the minimum of a negative intrinsic convex function, in a ball $B_{R}$ in the Heisenberg group, is controlled by the integral of the operator $H(u)$, where $H(u)$ is given by the sum of the determinant of the symmetric horizontal Hessian in the Heisenberg group, plus $\left(\partial_{t} u\right)^{2}$ times a constant. In that case, the approach to the subject is based on the idea that the Euclidean proof, see [18], might be repeated in the Heisenberg group, possibly by considering an intrinsic nice operator instead of the determinant of the Hessian matrix.

In that result, the absolute value of the minimum of any negative convex $C^{2}(\Omega) \cap C(\bar{\Omega})$ function, $\Omega \subset \mathbb{R}^{n}$ bounded, is controlled by the $L^{1}(\Omega)$ norm of the determinant of the Euclidean Hessian matrix of $u$. As far as we know, it is not evident if, as in the Euclidean case, see [18], from the inequality proved in [20] follows an inequality like the one we prove in this paper in Theorem 1.1, even if the results in [20] apply to a genuine class of nondivergence operators. Indeed, in [13], the authors prove that inequalities like the ones showed in [20] are not true without the additional term $\left(\partial_{t} u\right)^{2}$.

In the same paper, the authors also conjecture that the maximum of the absolute value of a convex intrinsic function in the Heisenberg group should be controlled by the $L^{Q}(\Omega)$ norm of the real part of the Kohn-Laplace operator. Indeed, in our Theorem 1.1, the $L^{Q}(\Omega)$ norm appears, see also [19].

Our approach follows essentially the idea contained in the paper [23] adapting Talenti's proof to the properties of the gauge distance in the Heisenberg group. Applications of Talenti's approach to some degenerate operators (with different types of degeneracy with respect to one treated here) are shown also in [1] and [14].

It is clear that our result is still far from solving the problem about the existence of a maximum principle for fully nonlinear operators modeled on the sub-Laplacian in the Heisenberg group. In any case, we think that the proof of Theorem 1.1 is interesting by itself, since it is based on intrinsic geometric tools (in particular the isoperimetric inequality in the Heisenberg group, see [21]), that permits to follow the scheme already successfully used in [23] for the Euclidean case (at least for the operators in divergence form). Hence, our result could be a useful step in the right direction. In the last Sect. 4, we show one of the cases where Theorem 1.1 can be applied, see Corollaries 4.1 and 4.2, to some nonlinear operators of concave/convex type that are not in divergence form. Eventually, concerning the fully nonlinear operators in Carnot groups and the existence of comparison results in literature, we point out also [5] and [6]. 


\section{Some preliminaries}

In the first subsection, we prove some preliminary results concerning the gauge distance. In the second one, we deal with rearrangements adapted to the framework of the Heisenberg group

2.1 Properties of the gauge distance

Let $(\xi, \eta, \sigma) \in \mathbb{H}^{n}$, then

$$
d_{G}(\xi, \eta, t)=\sqrt[4]{\left(|\xi|^{2}+|\eta|^{2}\right)^{2}+\sigma^{2}}=d_{K}((\xi, \eta, \sigma),(0,0,0)) .
$$

In particular, for every $i=1, \ldots, n$

$$
\begin{aligned}
X_{i} d_{G} & =\frac{1}{4}\left(\left(|\xi|^{2}+|\eta|^{2}\right)^{2}+\sigma^{2}\right)^{-\frac{3}{4}}\left(4\left(|\xi|^{2}+|\eta|^{2}\right) \xi_{i}+4 \sigma \eta_{i}\right) \\
& =\frac{1}{4} d_{G}^{-3}\left(4\left(|\xi|^{2}+|\eta|^{2}\right) \xi_{i}+4 \sigma \eta_{i}\right) \\
& =d_{G}^{-3}\left(\left(|\xi|^{2}+|\eta|^{2}\right) \xi_{i}+\sigma \eta_{i}\right)
\end{aligned}
$$

and

$$
\begin{aligned}
Y_{i} d_{G} & =\frac{1}{4}\left(\left(|\xi|^{2}+|\eta|^{2}\right)^{2}+t^{2}\right)^{-\frac{3}{4}}\left(4\left(|\xi|^{2}+|\eta|^{2}\right) \eta_{i}-4 \sigma \xi_{i}\right) \\
& =d_{G}^{-3}\left(\left(|\xi|^{2}+|\eta|^{2}\right) \eta_{i}-\sigma \xi_{i}\right) .
\end{aligned}
$$

Moreover, for every $i=1, \ldots, n$ :

$$
X_{i}^{2} d_{G}=-3 d_{G}^{-7}\left(\left(|\xi|^{2}+|\eta|^{2}\right) \xi_{i}+\sigma \eta_{i}\right)^{2}+d_{G}^{-3}\left(2 \xi_{i}^{2}+\left(|\xi|^{2}+|\eta|^{2}\right)+2 \eta_{i}^{2}\right)
$$

and

$$
Y_{i}^{2} d_{G}=-3 d_{G}^{-7}\left(\left(|\xi|^{2}+|\eta|^{2}\right) \eta_{i}-\sigma \xi_{i}\right)^{2}+d_{G}^{-3}\left(2 \eta_{i}^{2}+\left(|\xi|^{2}+|\eta|^{2}\right)+2 \xi_{i}^{2}\right) .
$$

As a consequence

$$
\begin{aligned}
\left|\nabla_{\mathbb{H}^{n}} d_{G}\right|^{2} & =\sum_{i=1}^{n}\left(\left(X_{i} d_{G}\right)^{2}+\left(Y_{i} d_{G}\right)^{2}\right)=d_{G}^{-6} \sum_{i=1}^{n}\left(\left(|\xi|^{2}+|\eta|^{2}\right)^{2}\left(\xi_{i}^{2}+\eta_{i}^{2}\right)+\sigma^{2}\left(\xi_{i}^{2}+\eta_{i}^{2}\right)\right) \\
& =d_{G}^{-6}\left(\left(|\xi|^{2}+|\eta|^{2}\right)^{3}+\sigma^{2}\left(|\xi|^{2}+|\eta|^{2}\right)\right)=\left(|\xi|^{2}+|\eta|^{2}\right) d_{G}^{-2},
\end{aligned}
$$

and

$$
\begin{aligned}
\Delta_{\mathbb{H}} d_{G} & =-3 d_{G}^{-7}\left(\left(|\xi|^{2}+|\eta|^{2}\right)^{3}+\sigma^{2}\left(|\xi|^{2}+|\eta|^{2}\right)\right)+(2 n+4) d_{G}^{-3}\left(|\xi|^{2}+|\eta|^{2}\right) \\
& =(2 n+1)\left(|\xi|^{2}+|\eta|^{2}\right) d_{G}^{-3} .
\end{aligned}
$$

In particular, we get the following result.

Lemma 2.1 Let $u$ be a solution of $\Delta_{\mathbb{H}^{n}} u=f$ in $\Omega \subseteq \mathbb{H}^{n}$. If $u \equiv u\left(d_{G}\right)$, then

$$
u^{\prime \prime}\left(d_{G}\right)+(2 n+1) \frac{u^{\prime}\left(d_{G}\right)}{d_{G}}=\frac{d_{G}^{2}}{\left(|\xi|^{2}+|\eta|^{2}\right)} f .
$$


Proof For every $i=1, \ldots, n$

$$
X_{i} u=u^{\prime}\left(d_{G}\right) X_{i} d_{G}, \text { and } Y_{i} u=u^{\prime}\left(d_{G}\right) Y_{i} d_{G} .
$$

Moreover for every $i=1, \ldots, n$

$$
X_{i}^{2} u=u^{\prime \prime}\left(d_{G}\right)\left(X_{i} d_{G}\right)^{2}+u^{\prime}\left(d_{G}\right) X_{i}^{2} d_{G}, \quad \text { and } Y_{i}^{2} u=u^{\prime \prime}\left(d_{G}\right)\left(Y_{i} d_{G}\right)^{2}+u^{\prime}\left(d_{G}\right) Y_{i}^{2} d_{G} .
$$

Thus,

$$
\Delta_{\mathbb{H}^{n}} u=u^{\prime \prime}\left(d_{G}\right)\left|\nabla u\left(d_{G}\right)\right|^{2}+u^{\prime}\left(d_{G}\right) \Delta_{\mathbb{H}^{n}} d_{G}=f\left(d_{G}\right) .
$$

In particular

$$
\Delta_{\mathbb{H}^{n}} u=u^{\prime \prime}\left(d_{G}\right)\left(|\xi|^{2}+|\eta|^{2}\right) d_{G}^{-2}+u^{\prime}\left(d_{G}\right)(2 n+4-3)\left(|\xi|^{2}+|\eta|^{2}\right) d_{G}^{-3}=f .
$$

Hence, we get

$$
u^{\prime \prime}\left(d_{G}\right)+(2 n+1) \frac{u^{\prime}\left(d_{G}\right)}{d_{G}}=\frac{d_{G}^{2}}{\left(|\xi|^{2}+|\eta|^{2}\right)} f
$$

ending the proof.

Let us denote by $B_{R}(0)$ the Koranyi ball centered in 0 , namely

$$
B_{R}(0)=\left\{(\xi, \eta, \sigma) \in \mathbb{H}^{n}: d_{G}(\xi, \eta, \sigma)<R\right\} .
$$

Lemma 2.2 Assume that $f(x)=\frac{|\xi|^{2}+|\eta|^{2}}{d_{G}^{2}(x)} g\left(d_{G}(x)\right)$, and suppose that

$$
\lim _{\rho \rightarrow 0} \rho g(\rho)=0 .
$$

Then, there exists a function $u \in C([0, R], \mathbb{R})$, such that $v=u\left(d_{G}\right)$ is solution of $\Delta_{\mathbb{H}^{n}} v=f$ in $B_{R}(0), v=0$ on $\partial B_{R}(0)$, and

$$
u(\rho)=-\int_{\rho}^{R} t^{-(2 n+1)}\left(\int_{0}^{t} \tau^{2 n+1} g(\tau) d \tau\right) d t,
$$

Proof Recalling the previous lemma, it is enough to find a solution of the following problem

$$
\left\{\begin{array}{l}
u^{\prime \prime}(\rho)+(2 n+1) \frac{u^{\prime}(\rho)}{\rho}=g(\rho) \\
u(R)=0
\end{array}\right.
$$

We are looking for a solution of the equation $u^{\prime \prime}(\rho)+(2 n+1) \frac{u^{\prime}(\rho)}{\rho}=g(\rho)$ in the form $\alpha(\rho) s^{2 n+1}$. We find that

$$
u(\rho)=u(0)+\int_{0}^{\rho} t^{-(2 n+1)}\left(\int_{0}^{t} \tau^{2 n+1} g(\tau) \mathrm{d} \tau\right) \mathrm{d} t .
$$

Notice that for every $\rho>0, u^{\prime}(\rho)=\rho^{-(2 n+1)} \int_{0}^{\rho} \tau^{2 n+1} g(\tau) \mathrm{d} \tau$. Thus,

$$
\lim _{\rho \rightarrow 0} u^{\prime}(\rho)=\lim _{\rho \rightarrow 0} \frac{\rho^{2 n+1} g(\rho)}{(2 n+1) \rho^{2 n}}=0 .
$$


As a consequence, $u$ is differentiable also in 0 and $u^{\prime}(0)=0$. If we impose that $u(R)=0$, then

$$
u(0)=-\int_{0}^{R} t^{-(2 n+1)}\left(\int_{0}^{t} \tau^{2 n+1} g(\tau) \mathrm{d} \tau\right) \mathrm{d} t .
$$

Thus, we get that

$$
u(\rho)=-\int_{\rho}^{R} t^{-(2 n+1)}\left(\int_{0}^{t} \tau^{2 n+1} g(\tau) \mathrm{d} \tau\right) \mathrm{d} t .
$$

Lemma 2.3 Assume that $v$ is the solution of the problem

$$
\begin{cases}\Delta_{\mathbb{H}^{n}} v=h, & B_{R}(0) \\ v=0, & \partial B_{R}(0),\end{cases}
$$

where $h=f \circ d_{G} \geq 0$. If $h \in L^{2 n+2}\left(B_{R}(0)\right)$, then

$$
\sup _{B_{R}(0)} v \leq C(n) R\|h\|_{L^{2 n+2}\left(B_{R}(0)\right)}
$$

Proof The function

$$
w(x)=u\left(d_{G}(x)\right)=-\int_{d_{G}(x)}^{R} t^{-(2 n+1)}\left(\int_{0}^{t} \tau^{2 n+1} f(\tau) \mathrm{d} \tau\right) \mathrm{d} t
$$

satisfies the problem

$$
\left\{\begin{array}{l}
u^{\prime \prime}(\rho)+(2 n+1) \frac{u^{\prime}(\rho)}{\rho}=f(\rho) \\
u(R)=0
\end{array}\right.
$$

Thus,

$$
u^{\prime \prime}\left(d_{G}(x)\right)+(2 n+1) \frac{u^{\prime}\left(d_{G}(x)\right)}{d_{G}(x)}=f\left(d_{G}(x)\right) \leq \frac{d_{G}^{2}}{|\xi|^{2}+|\eta|^{2}} f\left(d_{G}(x)\right),
$$

that is

$$
\frac{|\xi|^{2}+|\eta|^{2}}{d_{G}^{2}}\left(u^{\prime \prime}\left(d_{G}(x)\right)+(2 n+1) \frac{u^{\prime}\left(d_{G}(x)\right)}{d_{G}(x)}\right) \leq f\left(d_{G}(x)\right) .
$$

As a consequence, $w$ is a supersolution of $\Delta_{\mathbb{H}^{n}} v=h$. Hence, by the weak maximum principle,

$$
v \leq w
$$

in $B_{R}(0)$. We proceed to give an upper bound of $w$.

Concerning the definition of intrinsic perimeter in the Heisenberg group and its main application or characterization, we refer to [16] and [17]. For example, it was proved in [16] that for $C^{1}$ boundaries $\partial \Omega$,

$$
\mid \partial \Omega{\mid \mathbb{H}^{n}}=\sqrt{\sum_{i=1}^{n}\langle X, \eta\rangle_{E}^{2}+\sum_{i=1}^{n}\langle Y, \eta\rangle_{E}^{2}} \mathrm{~d} \mathcal{H}_{E}^{(2 n+1)}(\sigma)\llcorner\partial \Omega
$$


where $|\partial \Omega|_{\mathbb{H}^{n}}$, is the perimeter measure (see Proposition 2.14, p. 493 in [16], and Theorem 3.10, Corollary 3.11 in [17] for group of step 2), $\eta$ is the Euclidean unit normal vector to the surface $\partial \Omega$ and $\mathcal{H}_{E}^{(Q-2)}$ is the usual Hausdorff measure with respect to the Euclidean distance. Sometime, for the sake of simplicity, see [15], the spherical intrinsic Hausdorff measure is identified with the intrinsic Hausdorff measure itself. In any case here, we denote

$$
\mathcal{S}_{C C}^{(Q-1)}=\sqrt{\langle X, n\rangle_{E}^{2}+\langle Y, n\rangle_{E}^{2}} \mathcal{H}_{E}^{(Q-2)}\llcorner\partial \Omega,
$$

where $\mathcal{S}_{C C}^{(Q-1)}=\mathfrak{a} \tilde{\mathcal{S}}_{C C}^{(Q-1)}, \tilde{\mathcal{S}}_{C C}^{(Q-1)}$ is the spherical measure with respect to the CarnotCharathéodory distance and $\mathfrak{a}$ is a constant, see Proposition 2.14, p. 492 and Main Theorem p. 486 in [16]. Moreover, $\langle\cdot, \cdot\rangle_{E}$ denotes the usual inner product in $\mathbb{R}^{2 n+1}$. First, we notice that

$$
\tau^{2 n+1}=\bar{c}(1) \mathcal{S}_{C C}^{(Q-1)}\left(\partial \mathcal{B}_{\tau}(0)\right)
$$

where

$$
\mathcal{B}_{\tau}(0)=\left\{x \in \mathbb{H}^{n}: d(x, 0)<\tau\right\},
$$

$d(\cdot, \cdot)$ is the Carnot-Charathéodory distance in the Heisenberg group and $(\bar{c}(1))^{-1}$ $=\left|\partial \mathcal{B}_{1}(0)\right|_{\mathbb{H}^{n}}$. Thus, we can write, keeping in mind that $Q=2 n+2$ :

$$
\tau^{2 n+1}|f(\tau)|=\bar{c}(1) \int_{\partial \mathcal{B}_{\tau}(0)} \frac{|f(\gamma)|}{\left|\nabla_{\mathbb{H}^{n}} d(\gamma, 0)\right|} \mathrm{d} \mathcal{S}_{C C}^{(Q-1)}(\gamma)=\bar{c}(1) \int_{\partial \mathcal{B}_{\tau}(0)}|f(\gamma)| \mathrm{d} \mathcal{S}_{C C}^{(Q-1)}(\gamma) .
$$

As a consequence, recalling the coarea formula in the Heisenberg group, see e.g. [16], and that $d(\cdot, 0)$ satisfies the Eikonal equation, see [22], we get:

$$
\int_{0}^{t} \tau^{2 n+1}|f(\tau)| \mathrm{d} \tau=\bar{c}(1) \int_{\mathcal{B}_{t}(0)}|f(d(x, 0))| \mathrm{d} x .
$$

Then,

$$
\begin{aligned}
& w(x)=u\left(d_{G}(x)\right) \leq \int_{0}^{R} t^{-(2 n+1)}\left(\int_{0}^{t} \tau^{2 n+1}|f(\tau)| \mathrm{d} \tau\right) \mathrm{d} t \\
& \leq \bar{c}(1) \int_{0}^{R} t^{-(2 n+1)}\left(\int_{\mathcal{B}_{t}(0)}|f(d(x, 0))| \mathrm{d} x\right) \mathrm{d} t \\
& \leq \bar{c}(1) \int_{0}^{R} t^{-(2 n+1)}\left(\int_{\mathcal{B}_{t}(0)} \mathrm{d} x\right)^{\frac{1}{Q^{\prime}}}\left(\int_{\mathcal{B}_{t}(0)}|f(d(x, 0))|^{Q} \mathrm{~d} x\right)^{\frac{1}{Q}} \mathrm{~d} t \\
& =c(n) \int_{0}^{R} t^{-(2 n+1)} t^{\frac{Q}{Q^{\prime}}}\left(\int_{\mathcal{B}_{t}(0)}|f(d(x, 0))|^{Q} \mathrm{~d} x\right)^{\frac{1}{Q}} \mathrm{~d} t \\
& =c(n) \int_{0}^{R}\|f(d(x, 0))\|_{L^{Q}\left(\mathcal{B}_{t}(0)\right)} \mathrm{d} t \leq c(n) R\|f(d(x, 0))\|_{L^{Q}\left(\mathcal{B}_{R}(0)\right)} \text {, }
\end{aligned}
$$


where $Q^{\prime}=\frac{Q}{Q-1}=\frac{2 n+2}{2 n+1}$ and using the equivalence between Canot-Charatheodory distance and the gauge distance we get the result.

On the other hand, we have to point out that, see Proposition 5.4.4, p. 248 in [7],

$$
\int_{B_{t}(0)} f\left(d_{G}(x, 0)\right) \mathrm{d} x=Q \omega_{d_{G}} \int_{0}^{t} \tau^{Q-1} f(s) \mathrm{d} s,
$$

where $\omega_{d_{G}}$ is the Lebesgue measure of $B_{1}(0)$, the unit ball with respect to the gauge norm.

As a consequence, the result straightforwardly follows applying the Hölder inequality as before:

$$
\begin{aligned}
w(x) & =u\left(d_{G}(x)\right) \leq \int_{0}^{R} t^{-(2 n+1)}\left(\int_{0}^{t} \tau^{2 n+1}|f(\tau)| \mathrm{d} \tau\right) \mathrm{d} t \\
& \leq \frac{1}{Q \omega_{d_{G}}} \int_{0}^{R} t^{-(2 n+1)}\left(\int_{B_{t}(0)}\left|f\left(d_{G}(x, 0)\right)\right| \mathrm{d} x\right) \mathrm{d} t \\
& \leq \frac{1}{Q \omega_{d_{G}}} \int_{0}^{R} t^{-(2 n+1)}\left(\int_{B_{t}(0)} \mathrm{d} x\right)^{\frac{1}{Q^{\prime}}}\left(\int_{B_{t}(0)}\left|f\left(d_{G}(x, 0)\right)\right|^{Q} \mathrm{~d} x\right)^{\frac{1}{Q}} \mathrm{~d} t \\
& \left.=\frac{\omega_{d_{G}}^{\frac{1}{Q^{\prime}}}}{Q \omega_{d_{G}}} \int_{0}^{R} t^{-(2 n+1)} t^{\frac{Q}{Q^{\prime}}} \int_{B_{t}(0)}\left|f\left(d_{G}(x, 0)\right)\right|^{Q} \mathrm{~d} x\right)^{\frac{1}{Q}} \mathrm{~d} t \\
& =\frac{1}{Q \omega_{d_{G}}} \int_{0}^{R}\|f(d(x, 0))\|_{L^{Q}\left(B_{t}(0)\right)} \mathrm{d} t \leq \frac{1}{Q \omega_{d_{G}}^{\frac{1}{Q}}} R\left\|f\left(d_{G}(x, 0)\right)\right\|_{L} Q_{\left(B_{R}(0)\right)} .
\end{aligned}
$$

\subsection{Rearrangements}

The distribution function of a measurable function $u: \Omega \rightarrow \mathbb{R}$ is

$$
\mu(t)=|\{x \in \Omega:|u|>t\}|
$$

where, for every Lebesgue measurable set $A \subset \mathbb{R}^{n}$, we denote with $|A|$ the Lebesgue measure of $A$. The function $\mu$ is right continuous function of $t$, decreasing from $\mu(0)=1$ $\operatorname{supp} u \mid$ to $\lim _{t \rightarrow+\infty} \mu=0$ as $t$ increases from 0 to $+\infty$. The decreasing rearrangement of $u$ as the function $u^{*}:[0,+\infty] \rightarrow[0,+\infty]$ is defined as follows:

$$
u^{*}(s)=\inf \{t \geq 0: \mu(t)<s\} .
$$

If $\mu$ is strictly decreasing, $u^{*}$ is the decreasing function which extends to the whole of the half line $[0,+\infty]$ the inverse function of $\mu$. In particular, $u^{*}(0)=\operatorname{ess} \sup |u|$ and $u^{*}(|\Omega|)=0$. It is well known that

$$
\int_{\Omega}|u|^{p} \mathrm{~d} x=\int_{0}^{+\infty} u^{*}(t)^{p} \mathrm{~d} t,
$$


since both the integral equal $-\int_{0}^{+\infty}{ }_{t}^{p} \mu^{\prime}(t) \mathrm{d} t$. The classic Euclidean spherically symmetric rearrangement of $u$ is the function $u^{\sharp}: \mathbb{R}^{n} \rightarrow[0,+\infty]$ defined as

$$
u^{\sharp}(x)=u^{*}\left(\left|B_{E,|x|}(0)\right|\right),
$$

where $B_{E, t}(0)$ is the Euclidean ball centered in 0 of radius $t$ and $|x|$ is the usual Euclidean norm. For further definitions, we refer to [23].

In the sequel, we introduce a symmetric function that keeps into account the Heisenberg properties of the gauge ball. More precisely using, we define $u_{\mathbb{H}^{n}}^{\sharp}: \Omega_{\mathbb{H}^{n}}^{\sharp} \rightarrow \mathbb{R}$ as the symmetric rearrangement adapted to the Heisenberg group of the function $u: \Omega \rightarrow \mathbb{R}$ as follows: whenever $|\Omega|<+\infty$,

$$
u_{\mathbb{H}^{n}}^{\sharp}(x)=u^{*}\left(\left|B_{d_{G}(x)}^{\mathbb{H}^{n}}(0)\right|\right),
$$

where for every $0 \leq s \leq R_{\mathbb{H}^{n}}$

$$
\begin{aligned}
B_{s}^{\mathbb{H}^{n}}(0) & =\left\{x \in \mathbb{H}^{n}: d_{G}(x)<s\right\}, \\
\Omega_{\mathbb{H}^{n}}^{\sharp} & =B_{R_{\mathbb{H}^{n}}}^{\mathbb{H}^{n}}(0),
\end{aligned}
$$

and

$$
|\Omega|=\left|\Omega_{\mathbb{H}^{n}}^{\sharp}\right|=\left|\left\{x \in \mathbb{H}^{n}: d_{G}(x)<R_{\mathbb{H}^{n}}\right\}\right|=\left|B_{R_{\mathbb{H}^{n}}}^{\mathbb{H}^{n}}(0)\right|
$$

Obviously,

$$
\int_{\mathbb{H}^{n}}\left|u_{\mathbb{H}^{n}}^{\sharp}(x)\right|^{p} \mathrm{~d} x=\int_{0}^{+\infty} u^{*}(t)^{p} \mathrm{~d} t=\int_{\Omega}|u(x)|^{p} \mathrm{~d} x .
$$

As a consequence, $u_{\mathbb{H}^{n}}^{\sharp}$ is supported in $\Omega_{\mathbb{H}^{n}}^{\sharp}$ and

$$
\int_{\mathbb{H}^{n}}\left|u_{\mathbb{H}^{n}}^{\sharp}(x)\right|^{p} \mathrm{~d} x=\int_{\Omega_{\mathbb{H}^{n}}^{\sharp}}\left|u_{\mathbb{H}^{n}}^{\sharp}(x)\right|^{p} \mathrm{~d} x=\int_{\Omega}|u(x)|^{p} \mathrm{~d} x .
$$

Moreover,

$$
\int_{\Omega}|u v| \mathrm{d} x \leq \int_{0}^{+\infty} u^{*}(s) v^{*}(s) \mathrm{d} s .
$$

On the other hand,

$$
\begin{aligned}
\int_{\mathbb{H}^{n}} u_{\mathbb{H}^{n}}^{\sharp}(x) v_{\mathbb{H}^{n}}^{\sharp}(x) \mathrm{d} x & =\int_{0}^{+\infty}\left|\left\{x: u^{*}\left(\left|B_{d_{G}(x)}^{\mathbb{H}^{n}}(0)\right|\right) v^{*}\left(\left|B_{d_{G}(x)}^{\mathbb{H}^{n}}(0)\right|\right)>s\right\}\right| \mathrm{d} s \\
& =\int_{0}^{+\infty} u^{*}(s) v^{*}(s) \mathrm{d} s .
\end{aligned}
$$

Thus,

$$
\int_{\Omega}|u v| \mathrm{d} x \leq \int_{\mathbb{H}^{n}} u_{\mathbb{H}^{n}}^{\sharp}(x) v_{\mathbb{H}^{n}}^{\sharp}(x) \mathrm{d} x .
$$




\section{Proof of the main results}

3.1 The real part of the Kohn-Laplace operator case

We recall that we denote

$$
\mathcal{S}_{C C}^{(Q-1)}=\sqrt{\sum_{i=1}^{n}\left\langle X_{i}, \eta\right\rangle_{E}^{2}+\sum_{i=1}^{n}\left\langle Y_{i}, \eta\right\rangle_{E}^{2}} \mathcal{H}_{E}^{(Q-2)}\llcorner\partial \Omega,
$$

where $\eta$ is the Euclidean unit normal vector to the surface $\partial \Omega$. Let $u$ be the solution of $\Delta_{\mathbb{H}^{n}} u=f$ in $\Omega$ with $u=0$ on $\partial \Omega$. Then, denoting $v=\frac{\nabla_{\mathbb{H}} n u(x)}{\left|\nabla_{\mathbb{H}^{n}} u(x)\right|}$

$$
\int_{u>t} \Delta_{\mathbb{H}^{n}} u \mathrm{~d} x=\int_{\{u=t\}}\left\langle\nabla_{\mathbb{H}^{n}} u, v\right\rangle_{\mathbb{H}^{n}} \mathrm{~d} \mathcal{S}_{C C}^{(Q-1)}(\gamma)=\int_{\{u=t\}}\left|\nabla_{\mathbb{H}^{n}} u\right| d \mathcal{S}_{C C}^{(Q-1)}(\gamma)
$$

As a consequence

$$
\int_{u>t} f(x) \mathrm{d} x=\int_{u>t} \Delta_{\mathbb{H}^{n}} u \mathrm{~d} x=\int_{\{u=t\}}\left|\nabla_{\mathbb{H}^{n}} u\right| \mathrm{d} \mathcal{S}_{C C}^{(Q-1)}(\gamma) .
$$

Keeping in mind the coarea formula in the Heisenberg group, it results

$$
-\mu^{\prime}(t)=\int_{\{u=t\}} \frac{1}{\left|\nabla_{\mathbb{H}^{n}} u\right|} \mathrm{d} \mathcal{S}_{C C}^{(Q-1)}(\gamma) .
$$

Thus, by Schwarz inequality,

$$
\begin{aligned}
|\{u=t\}|_{\mathbb{H}^{n}} & \leq\left(\int_{\{u=t\}} \frac{1}{\left|\nabla_{\mathbb{H}^{n}} u\right|} \mathrm{d} \mathcal{S}_{C C}^{(Q-1)}(\gamma)\right)^{\frac{1}{2}}\left(\int_{\{u=t\}}\left|\nabla_{\mathbb{H}^{n}} u\right| \mathrm{d} \mathcal{S}_{C C}^{(Q-1)}(\gamma)\right)^{\frac{1}{2}} \\
& =\left(-\mu^{\prime}(t)\right)^{\frac{1}{2}}\left(\int_{\{u=t\}}\left|\nabla_{\mathbb{H}^{n}} u\right| \mathrm{d} \mathcal{S}_{C C}^{(Q-1)}(\gamma)\right)^{\frac{1}{2}} .
\end{aligned}
$$

Now recalling the isoperimetric inequality in the Heisenberg group, see [21], we get

$$
|\{u=t\}| \mathbb{H}^{n} \geq K_{\mathbb{H}}|\{u>t\}|^{1-\frac{1}{Q}} .
$$

Hence,

$$
\int_{\{u=t\}}\left|\nabla_{\mathbb{H}^{n}} u\right| \mathrm{d} \mathcal{S}_{C C}^{(Q-1)}(\gamma) \geq K_{\mathbb{H}}^{2}\left(-\mu^{\prime}(t)\right)^{-1}|\{u>t\}|^{2-\frac{2}{Q}}
$$

thus, keeping in mind, (11) we get

$$
\int_{u>t} f(x) \mathrm{d} x \geq K_{\mathbb{H}}^{2}\left(-\mu^{\prime}(t)\right)^{-1}|\{u>t\}|^{2-\frac{2}{Q}} .
$$


Now, recalling (10), we get that

$$
\int_{0}^{\mu(t)} f^{*}(s) \mathrm{d} s \geq K_{\mathbb{H}}^{2}\left(-\mu^{\prime}(t)\right)^{-1}|\{u>t\}|^{2-\frac{2}{Q}}
$$

that it is equivalent to write

$$
\int_{0}^{\mu(t)} f^{*}(s) \mathrm{d} s K_{\mathbb{H}}^{-2}\left(-\mu^{\prime}(t)\right)|\{u>t\}|^{-2+\frac{2}{Q}} \geq 1 .
$$

Integrating in $[0, t]$, we get

$$
K_{\mathbb{H}}^{-2} \int_{0}^{t}\left(\int_{0}^{\mu(r)} f^{*}(s) \mathrm{d} s\left(-\mu^{\prime}(t)\right)|\{u>t\}|^{-2+\frac{2}{Q}}\right) \mathrm{d} r \geq t
$$

from which follows

$$
-K_{\mathbb{H}}^{-2} \int_{\mu(t)}^{|\Omega|} r^{-2+\frac{2}{Q}}\left(\int_{0}^{r} f^{*}(s) \mathrm{d} s\right) \mathrm{d} r \geq t
$$

that implies

$$
-K_{\mathbb{H}}^{-2} \int_{\tau}^{|\Omega|} r^{-2+\frac{2}{Q}}\left(\int_{0}^{r} f^{*}(s) \mathrm{d} s\right) \mathrm{d} r \geq u^{*}(\tau) .
$$

Hence,

$$
-K_{\mathbb{H}}^{-2} \int_{\left|B_{d_{G}(x)}(0)\right|}^{|\Omega|} r^{-2+\frac{2}{Q}}\left(\int_{0}^{r} f^{*}(s) \mathrm{d} s\right) \mathrm{d} r \geq u_{\mathbb{H}^{n}}^{\sharp}(x) .
$$

Notice that $\left|B_{d_{G}(x)}(0)\right|=\omega_{d_{G}}\left|d_{G}(x)\right|^{Q}$. Hence, supposing to make the change of variables $\omega_{d_{G}}|t|^{Q}=r$, we get

$$
\begin{aligned}
\int_{\left|B_{d_{G}(x)}(0)\right|}^{|\Omega|} r^{-2+\frac{2}{Q}}\left(\int_{0}^{r} f^{*}(s) \mathrm{d} s\right) \mathrm{d} r & =\int_{d_{G}(x)}^{R}\left(\omega_{d_{G}} t^{Q}\right)^{-2+\frac{2}{Q}}\left(\int_{0}^{\omega_{d_{G}} t^{Q}} f^{*}(s) \mathrm{d} s\right) Q \omega_{d_{G}} t^{Q-1} \mathrm{~d} t \\
& =\int_{d_{G}(x)}^{R}\left(\omega_{d_{G}} t\right)^{-2 Q+2}\left(\int_{0}^{\omega_{d_{G}} t^{Q}} f^{*}(s) \mathrm{d} s\right) Q \omega_{d_{G}} t^{Q-1} \mathrm{~d} t
\end{aligned}
$$




$$
\begin{aligned}
& =Q \omega_{d_{G}}^{-2 Q+3} \int_{d_{G}(x)}^{R} t^{-Q+1}\left(\int_{0}^{\omega_{d_{G}} t^{Q}} f^{*}(s) \mathrm{d} s\right) \mathrm{d} t \\
& =Q \omega_{d_{G}}^{-2 Q+3} \int_{d_{G}(x)}^{R} t^{-Q+1}\left(\int_{B_{t}(0)} f^{\sharp}(x) \mathrm{d} x\right) \mathrm{d} t .
\end{aligned}
$$

Moreover, by the change of variables $s=\omega_{d_{G}} \tau^{Q}$ in the second integral

$$
\begin{aligned}
& Q \omega_{d_{G}}^{-2 Q+3} \int_{d_{G}(x)}^{R} t^{-Q+1}\left(\int_{B_{t}(0)} f^{\sharp}(x) \mathrm{d} x\right) \mathrm{d} t=Q \omega_{d_{G}}^{-2 Q+3} \int_{d_{G}(x)}^{R} t^{-Q+1}\left(\int_{0}^{\omega_{d_{G}} t^{Q}} f^{*}(s) \mathrm{d} s\right) \mathrm{d} t \\
& =Q \omega_{d_{G}}^{-2 Q+3} \int_{d_{G}(x)}^{R} t^{-Q+1}\left(\int_{0}^{t} Q \omega_{d_{G}} \tau^{Q-1} f^{*}\left(\omega_{d_{G}} \tau^{Q}\right) \mathrm{d} s\right) \mathrm{d} \tau \\
& =Q^{2} \omega_{d_{G}}^{-2 Q+4} \int_{d_{G}(x)}^{R} t^{-Q+1}\left(\int_{0}^{t} \tau^{Q-1} f^{*}\left(\omega_{d_{G}} \tau^{Q}\right) \mathrm{d} s\right) \mathrm{d} \tau .
\end{aligned}
$$

We already know that the function $w(x)=-\int_{d_{G}(x)}^{R} t^{-Q+1}\left(\int_{0}^{t} \tau^{Q-1} f^{*}\left(\omega_{d_{G}} \tau^{Q}\right) \mathrm{d} s\right) \mathrm{d} \tau$ is a supersolution of

$$
\Delta_{\mathbb{H}^{n}} v=f_{\mathbb{H}}^{\sharp}, \quad \Omega_{\mathbb{H}^{n}}^{\sharp}
$$

and moreover

$$
\begin{cases}\Delta_{\mathbb{H}^{n}} v=f_{\mathbb{H}}^{\sharp}, & \Omega_{\mathbb{H}^{n}}^{\sharp} \\ v=0, & \partial \Omega_{\mathbb{H}^{n}}^{\sharp}\end{cases}
$$

Hence, we can summarize by writing that

$$
K_{\mathbb{H}^{n}}^{2} Q^{2} \omega_{d_{G}}^{-2 Q+4} w(x) \geq u_{\mathbb{H}^{n}}^{\sharp}(x) .
$$

In particular,

$$
\frac{K_{\mathbb{H}^{n}}^{2} Q}{2 Q-4+\frac{1}{Q}} R\|f\|_{L^{Q}\left(B_{R}(0)\right)} \geq K_{\mathbb{H}^{n}}^{2} Q^{2} \omega_{d_{G}}^{-2 Q+4} w(x) \geq u_{\mathbb{H}^{n}}^{\sharp}(x) .
$$

Corollary 3.1 Let $\Omega \subset \mathbb{H}^{n}$ be a open bounded set and $f \in L^{Q}(\Omega)$. If $u$ is a $C(\bar{\Omega}) \cap C^{2}(\Omega)$ solution of

$$
\Delta_{\mathbb{H}^{n}} u=f
$$

in $\Omega$, then there exists a positive constant $C(Q), Q=2 n+2$ such that

$$
\sup _{\Omega} u \leq \sup _{\partial \Omega} u+C(Q)|\Omega|^{\frac{1}{Q}}\|f\|_{L^{Q}(\Omega)} .
$$


Proof Let $v=u-\sup _{\partial \Omega} u$. Then, $v \leq 0$ on $\partial \Omega$. By the maximum principle, $v \leq w$ where $\Delta_{\mathbb{H}^{n}} w=f$ and $w=0$ on $\partial \Omega$. As a consequence

$$
\sup _{\Omega} v \leq \sup _{\Omega} w \leq C(Q)|\Omega|^{\frac{1}{Q}}\|f\|_{L^{Q}(\Omega)} .
$$

Moreover

$$
\sup _{\Omega} v=\sup _{\Omega} u-\sup _{\partial \Omega} u
$$

so that

$$
\sup _{\Omega} u \leq \sup _{\partial \Omega} u+C(Q)|\Omega|^{\frac{1}{Q}}\|f\|_{L^{Q}(\Omega)}
$$

\subsection{The case of degenerate operators in divergence form}

Let $A$ be a symmetric $2 n \times 2 n$ positive definite matrix. Let $\nabla_{\mathbb{H}^{n}} u$ be the horizontal gradient.

If $u$ is the solution of

$$
\operatorname{div}_{\mathbb{H}^{n}}\left(A(x) \nabla_{\mathbb{H}^{n}} u(x)\right)=f
$$

in $\Omega$, with $u=0$ on $\partial \Omega$, then denoting as usual $v=\frac{\nabla_{\mathbb{H}^{n}} u(x)}{\left|\nabla_{\mathbb{H}^{n}} u(x)\right|}$ we get

$$
\begin{aligned}
& \int_{u>\kappa} \operatorname{div}_{\mathbb{H}^{n}}\left(A(x) \nabla_{\mathbb{H}^{n}} u(x)\right) \mathrm{d} x \\
& \quad=\int_{\{u=\kappa\}}\left\langle A(x) \nabla_{\mathbb{H}^{n}} u, v\right\rangle_{\mathbb{H}^{n}} d \mathcal{S}_{C C}^{(Q-1)}(\gamma) \geq \lambda \int_{\{u=t\}}\left|\nabla_{\mathbb{H}^{n}} u\right| \mathrm{d} \mathcal{S}_{C C}^{(Q-1)}(\gamma) .
\end{aligned}
$$

As a consequence

$$
\int_{u>t} f(x) \mathrm{d} x=\int_{u>\kappa} \operatorname{div}_{\mathbb{H}^{n}}\left(A(x) \nabla_{\mathbb{H}^{n}} u(x)\right) \mathrm{d} x \geq \lambda \int_{\{u=\kappa\}}\left|\nabla_{\mathbb{H}^{n}} u\right| \mathrm{d} \mathcal{S}_{C C}^{(Q-1)}(\gamma) .
$$

Keeping in mind the coarea formula in the Heisenberg group, it results

$$
-\mu^{\prime}(\kappa)=\int_{\{u=\kappa\}} \frac{1}{\left|\nabla_{\mathbb{H}^{n}} u\right|} \mathrm{d} \mathcal{S}_{C C}^{(Q-1)}(\mathrm{d} \gamma) .
$$

Thus, by Schwarz inequality,

$$
\begin{aligned}
|\{u=\kappa\}| \mathbb{H}^{n} & \leq\left(\int_{\{u=\kappa\}} \frac{1}{\left|\nabla_{\mathbb{H}^{n}} u\right|} \mathrm{d} \mathcal{S}_{C C}^{(Q-1)}(\gamma)\right)^{\frac{1}{2}}\left(\int_{\{u=\kappa\}}\left|\nabla_{\mathbb{H}^{n}} u\right| \mathrm{d} \mathcal{S}_{C C}^{(Q-1)}(\gamma)\right)^{\frac{1}{2}} \\
& =\left(-\mu^{\prime}(\kappa)\right)^{\frac{1}{2}}\left(\int_{\{u=\kappa\}}\left|\nabla_{\mathbb{H}^{n}} u\right| \mathrm{d} \mathcal{S}_{C C}^{(Q-1)}(\gamma)\right)^{\frac{1}{2}} .
\end{aligned}
$$

Now recalling the isoperimetric inequality we get

$$
|\{u=\kappa\}|_{\mathbb{H}^{n}} \geq K_{\mathbb{H}}|\{u>\kappa\}|^{1-\frac{1}{Q}} .
$$


Hence,

$$
\lambda \int_{\{u=\kappa\}}\left|\nabla_{\mathbb{H}^{n}} u\right| \mathrm{d} \mathcal{S}_{C C}^{(Q-1)}(\gamma) \geq \lambda K_{\mathbb{H}}^{2}\left(-\mu^{\prime}(\kappa)\right)^{-1}|\{u>\kappa\}|^{2-\frac{2}{Q}}
$$

thus, keeping in mind, (19) we get

$$
\int_{u>\kappa} f(x) \mathrm{d} x \geq \lambda K_{\mathbb{H}}^{2}\left(-\mu^{\prime}(\kappa)\right)^{-1}|\{u>\kappa\}|^{2-\frac{2}{Q}} .
$$

Now, recalling (10), we get that

$$
\int_{0}^{\mu(\kappa)} f^{*}(s) \mathrm{d} s \geq \lambda K_{\mathbb{H}}^{2}\left(-\mu^{\prime}(\kappa)\right)^{-1}|\{u>\kappa\}|^{2-\frac{2}{Q}}
$$

that it is equivalent to write

$$
\lambda^{-1} K_{\mathbb{H}}^{-2} \int_{0}^{\mu(\kappa)} f^{*}(s) \mathrm{d} s\left(-\mu^{\prime}(\kappa)\right)|\{u>\kappa\}|^{-2+\frac{2}{Q}} \geq 1 .
$$

Integrating in $[0, \kappa]$, we get

$$
\lambda^{-1} K_{\mathbb{H}}^{-2} \int_{0}^{\kappa}\left(\int_{0}^{\mu(r)} f^{*}(s) \mathrm{d} s\left(-\mu^{\prime}(\kappa)\right)|\{u>\kappa\}|^{-2+\frac{2}{Q}}\right) \mathrm{d} r \geq \kappa
$$

from which follows

$$
-\lambda^{-1} K_{\mathbb{H}}^{-2} \int_{\mu(\kappa)}^{|\Omega|} r^{-2+\frac{2}{Q}}\left(\int_{0}^{r} f^{*}(s) \mathrm{d} s\right) \mathrm{d} r \geq \kappa
$$

that implies

$$
-\lambda^{-1} K_{\mathbb{H}}^{-2} \int_{\tau}^{|\Omega|} r^{-2+\frac{2}{Q}}\left(\int_{0}^{r} f^{*}(s) \mathrm{d} s\right) \mathrm{d} r \geq u^{*}(\tau)
$$

Hence,

$$
-\lambda^{-1} K_{\mathbb{H}}^{-2} \int_{\left|B_{d_{G}(x)}(0)\right|}^{|\Omega|} r^{-2+\frac{2}{Q}}\left(\int_{0}^{r} f^{*}(s) \mathrm{d} s\right) \mathrm{d} r \geq u_{\mathbb{H}^{n}}^{\sharp}(x) .
$$


Notice that $\left|B_{d_{G}(x)}(0)\right|=\omega_{d_{G}}\left|d_{G}(x)\right|^{Q}$. Hence, supposing to make the change of variables $\omega_{d_{G}}|\kappa|^{Q}=r$, we get

$$
\begin{aligned}
& \int_{\mid B_{d_{G}}(x)}^{|\Omega|} r^{-2+\frac{2}{Q}}\left(\int_{0}^{r} f^{*}(s) \mathrm{d} s\right) \mathrm{d} r \\
= & \int_{d_{G}(x)}^{R}\left(\omega_{d_{G}} \kappa^{Q}\right)^{-2+\frac{2}{Q}}\left(\int_{0}^{\omega_{d_{G}} \kappa^{Q}} f^{*}(s) \mathrm{d} s\right) Q \omega_{d_{G}} \kappa^{Q-1} \mathrm{~d} \kappa \\
= & \int_{d_{G}(x)}^{R}\left(\omega_{d_{G}} \kappa\right)^{-2 Q+2}\left(\int_{0}^{\omega_{d_{G}} \kappa^{Q}} f^{*}(s) \mathrm{d} s\right) Q \omega_{d_{G}} \kappa^{Q-1} \mathrm{~d} \kappa \\
= & Q \omega_{d_{G}}^{-2 Q+3} \int_{d_{G}(x)}^{R} \kappa^{-Q+1}\left(\int_{0}^{\omega_{d_{G}} \kappa^{Q}} f^{*}(s) \mathrm{d} s\right) \mathrm{d} \kappa \\
= & Q \omega_{d_{G}}^{-2 Q+3} \int_{d_{G}(x)}^{R} \kappa^{-Q+1}\left(\int_{B_{\kappa}(0)}^{R} f^{\sharp}(x) \mathrm{d} x\right) \mathrm{d} \kappa .
\end{aligned}
$$

Moreover, by the change of variables $s=\omega_{d_{G}} \tau$ in the second integral

$$
\begin{aligned}
& Q \omega_{d_{G}}^{-2 Q+3} \int_{d_{G}(x)}^{R} \kappa^{-Q+1}\left(\int_{B_{\kappa}(0)} f^{\sharp}(x) \mathrm{d} x\right) \mathrm{d} \kappa \\
& =Q \omega_{d_{G}}^{-2 Q+3} \int_{d_{G}(x)}^{R} \kappa^{-Q+1}\left(\int_{0}^{\omega_{d_{G}} \kappa^{Q}} f^{*}(s) \mathrm{d} s\right) \mathrm{d} \kappa \\
& =Q \omega_{d_{G}}^{-2 Q+3} \int_{d_{G}(x)}^{R} \kappa^{-Q+1}\left(\int_{0}^{\kappa} Q \omega_{d_{G}} \tau^{Q-1} f^{*}\left(\omega_{d_{G}} \tau^{Q}\right) \mathrm{d} \tau\right) \mathrm{d} \kappa \\
& =Q^{2} \omega_{d_{G}}^{-2 Q+4} \int_{d_{G}(x)}^{R} \kappa^{-Q+1}\left(\int_{0}^{\kappa} \tau^{Q-1} f^{*}\left(\omega_{d_{G}} \tau^{Q}\right) \mathrm{d} \tau\right) \mathrm{d} \kappa .
\end{aligned}
$$

We already know that the function $w(x)=-\int_{d_{G}(x)}^{R} \kappa^{-Q+1}\left(\int_{0}^{\kappa} \tau^{Q-1} f^{*}\left(\omega_{d_{G}} \tau^{Q}\right) \mathrm{d} \tau\right) \mathrm{d} \kappa$ is a supersolution of

$$
\Delta_{\mathbb{H}^{n}} v=f_{\mathbb{H}}^{\sharp}, \quad \Omega_{\mathbb{H}^{n}}^{\sharp}
$$

and moreover

$$
\begin{cases}\Delta_{\mathbb{H}^{n}} v=f_{\mathbb{H}}^{\sharp}, & \Omega_{\mathbb{H}^{n}}^{\sharp} \\ v=0, & \partial \Omega_{\mathbb{H}^{n}}^{\sharp}\end{cases}
$$


Hence, we can resume writing that

$$
\lambda^{-1} K_{\mathbb{H}^{n}}^{2} Q^{2} \omega_{d_{G}}^{-2 Q+4} w(x) \geq u_{\mathbb{H}^{n}}^{\sharp}(x) .
$$

In particular,

$$
\lambda^{-1} \frac{K_{\mathbb{H}^{n}}^{2} Q}{2 Q-4+\frac{1}{Q}} R\|f\|_{L^{Q}\left(B_{R}(0)\right)} \geq K_{\mathbb{H}^{n}}^{2} Q^{2} \omega_{d_{G}}^{-2 Q+4} w(x) \geq u_{\mathbb{H}^{n}}^{\sharp}(x) .
$$

concluding the proof of the first part of Theorem 1.1. Thus, we can state the following result.

Moreover, let us remark that for every $2 n \times 2 n$ matrix $A$, with constant coefficients, it results

$$
\operatorname{div}_{\mathbb{H}^{n}}\left(A \nabla_{\mathbb{H}^{n}} u(x)\right)=\mathcal{L}_{\mathbb{H}^{n}, A} u=\operatorname{Tr}\left(A D_{\mathbb{H}^{n}}^{2} u\right) .
$$

In particular, if $A=A^{T}$ and its coefficients are constant, then

$$
\operatorname{div}_{\mathbb{H}^{n}}\left(A \nabla_{\mathbb{H}^{n}} u(x)\right)=\mathcal{L}_{\mathbb{H}^{n}, A} u=\operatorname{Tr}\left(A D_{\mathbb{H}^{n}}^{2 *} u\right),
$$

where $D_{\mathbb{H}^{n}}^{2 *} u$ is the horizontal Hessian matrix of $u$.

Let $u \in C^{2}(\Omega) \cap C(\bar{\Omega})$. Then, for every constant matrix $A$

$$
\operatorname{div}_{\mathbb{H}^{n}}\left(A \nabla_{\mathbb{H}^{n}} u(x)\right)=\operatorname{Tr}\left(A D_{\mathbb{H}^{n}}^{2} u\right) .
$$

Thus, we can apply the previous result to $f=\operatorname{Tr}\left(A D_{\mathbb{H}^{n}}^{2} u\right)$, obtaining:

$$
\sup _{\Omega} u \leq \sup _{\partial \Omega} u+C(n, \lambda)|\Omega|^{\frac{1}{Q}}\left\|\operatorname{Tr}\left(A D_{\mathbb{H}^{n}}^{2} u\right)\right\|_{L^{Q}(\Omega)} .
$$

\section{Some applications to some nonlinear operators}

Let $\Omega \subseteq \mathbb{R}^{2 n+1}$ be an open nonempty set. Let $F: S^{2 n \times 2 n} \times \Omega \rightarrow \mathbb{R}$ be a function such that for any $M \in S^{2 n \times 2 n}$ and for every $x \in \Omega \subset \mathbb{R}^{2 n+1}$

$$
\lambda\|N\| \leq F(M+N, x)-F(M, x) \leq \Lambda\|N\|,
$$

whenever $N \geq 0$, and $\|M\|=\sup _{|v|=1, v \in \mathbb{R}^{2 n}}|M v|$, where $S^{2 n \times 2 n}$ denotes the set of symmetric square matrices of order $2 n$ and $0<\lambda \leq \Lambda$ are constants. If $F$ satisfies the previous assumption and $D_{\mathbb{H}^{n}}^{2} u$ is the symmetric horizontal Hessian matrix in the Heisenberg group, we say that $F\left(D_{\mathbb{H}^{n}}^{2} \cdot x\right)$ is a fully nonlinear uniformly horizontal degenerate elliptic operator.

We are interested in the existence of a priori estimate of the solutions of the equation

$$
F\left(D_{\mathbb{H}^{n}}^{2} u(x), x\right)=f(x),
$$

in $\Omega$ bounded and $f: \Omega \rightarrow \mathbb{R}$ is a given function.

If $F(\cdot)$ is the trace of the matrix $M$, and $D_{\mathbb{H}^{n}}^{2 *} u$ denotes the horizontal Hessian matrix in the Heisenberg group, see below for the definition, then $F\left(D_{\mathbb{H}^{n}}^{2 *} u\right)=\operatorname{Tr}\left(D_{\mathbb{H}^{n}}^{2 *} u\right)=\Delta_{\mathbb{H}^{n}} u$, that is, we get the real part of the Kohn-Laplace operator in the Heisenberg group.

Thus, we just remark that, even the definition of fully nonlinear degenerate elliptic operator we gave is very similar to the well-known definition of nonlinear uniformly elliptic operator, see [11], it is worth pointing out that in the above definition, the matrices belong to $S^{2 n \times 2 n}$ although $\Omega$ is a subset of $\mathbb{H}^{n} \equiv \mathbb{R}^{2 n+1}$. 
Let $0<\lambda \leq \Lambda$ be real numbers. We define

$$
\mathcal{A}_{\lambda, \Lambda}=\left\{A \in S^{2 n \times 2 n}: \lambda \sqrt{\sum_{i=1}^{2 n} v_{i}^{2}} \leq \sum_{i, j=1}^{2 n} a_{i j} v_{i} v_{j} \leq \Lambda \sqrt{\sum_{i=1}^{2 n} v_{i}^{2}}, \quad v \in \mathbb{R}^{2 n}\right\} .
$$

The simplest example of horizontally degenerate uniformly elliptic fully nonlinear operators is the following extremal operators:

$$
\mathcal{P}_{\mathbb{H}^{n}}^{+}(u)(x)=\sup _{A \in \mathcal{A}_{\lambda, \Lambda}} \operatorname{Tr}\left(A D_{\mathbb{H}^{n}}^{2 *} u(x)\right) ; \quad \mathcal{P}_{\mathbb{H}^{n}}^{-}(u)(x)=\inf _{A \in \mathcal{A}_{\lambda, \Lambda}} \operatorname{Tr}\left(A D_{\mathbb{H}^{n}}^{2 *} u(x)\right) .
$$

$\mathcal{P}^{+}$and $\mathcal{P}^{-}$are, respectively, examples of convex and concave operators.

4.1 Properties of degenerate nonlinear uniformly elliptic operators

We shall say that $F$ is an horizontal concave operator, if for every symmetric matrices $M, N \in S^{2 n \times 2 n}$, for every $x \in \Omega \subset \mathbb{H}^{n}$, and for every $s \in[0,1]$

$$
F(t M+(1-s) N) \geq s F(M)+(1-s) F(N) .
$$

Analogously we shall say that $F$ an horizontal convex operator, if for every symmetric matrices $M, N \in S^{2 n \times 2 n}$, for every $x \in \Omega \subset \mathbb{H}^{n}$, and for every $s \in[0,1]$

$$
F(t M+(1-s) N) \leq s F(M)+(1-s) F(N) .
$$

If $F$ is degenerate nonlinear uniformly elliptic operators of class $C^{1}$, that is, concave and $F(0)=0$, then there exists a matrix $B_{0} \in \mathcal{A}_{\lambda, \Lambda}$ such that

$$
F(M) \leq \operatorname{Tr}\left(B_{0} M\right) .
$$

If $F$ is degenerate nonlinear uniformly elliptic operators of class $C^{1}$, that is, concave and $F(0)=0$, then there exists a matrix $B_{0} \in \mathcal{A}_{\lambda, \Lambda}$ such that

$$
F(M) \geq \operatorname{Tr}\left(B_{0} M\right) .
$$

Notice that if $F$ is convex, then $-F$ is concave, and if $F$ is concave, then $-F$ is convex. We point out that $\mathcal{P}^{+}$is convex and $\mathcal{P}^{-}$is concave. Moreover, $-\mathcal{P}^{+}$is concave and $-\mathcal{P}^{-}$is convex and

$$
\mathcal{P}^{+}(M)=-\mathcal{P}^{-}(-M), \quad \mathcal{P}^{-}(M)=-\mathcal{P}^{+}(-M)
$$

4.2 Some partial results: concave/convex operators

Corollary 4.1 Let $F: S^{2 n} \times \Omega \rightarrow \mathbb{R}$ be a horizontal degenerate elliptic fully nonlinear operator. If there exists a matrix $B_{0} \in \mathcal{A}_{\lambda, \Lambda}$ such that for every $M \in S^{2 n}$

$$
F(M, x) \leq \operatorname{Tr}\left(B_{0} M\right),
$$

then if $u \in C^{2}(\Omega) \cap C(\bar{\Omega})$ is solution of

$$
F\left(D^{2 *} u(x), x\right) \geq f(x), \Omega
$$

and $f \in L^{Q}(\Omega)$, then

$$
\sup _{\Omega} u \leq \sup _{\partial \Omega} u+C(n, \lambda)|\Omega|^{\frac{1}{Q}}\|f\|_{L} Q_{(\Omega)} .
$$


As a consequence, we can prove the following theorem that is a corollary of previous Corollary 4.1 .

Corollary 4.2 Let $\Omega \subset \mathbb{H}^{n}$ be a bounded set. Let $F: S^{2 n \times 2 n} \rightarrow \mathbb{R}$ be a concave fully nonlinear uniformly horizontally degenerate elliptic operator such that $F(0)=0$. If $u \in$ $C^{2}(\Omega) \cap C(\bar{\Omega})$ is a solution of

$$
F\left(D_{\mathbb{H}^{n}}^{2 *} u(x)\right) \geq f(x),
$$

and $f \in L^{Q}(\Omega)$, then

$$
\sup _{\Omega} u \leq \sup _{\partial \Omega} u+C(n, \lambda)|\Omega|^{\frac{1}{Q}}\|f\|_{L^{Q}(\Omega)} .
$$

Corollary 4.3 Let $\Omega \subset \mathbb{H}^{n}$ be a bounded set. Let $F: S^{2 n \times 2 n} \rightarrow \mathbb{R}$ be a convex fully nonlinear uniformly horizontally degenerate elliptic operator such that $F(0)=0$. If $u \in$ $C^{2}(\Omega) \cap C(\bar{\Omega})$ is a solution of

$$
F\left(D_{\mathbb{H}^{n}}^{2 *} u(x)\right) \leq f(x)
$$

and $f \in L^{Q}(\Omega)$, then

$$
\sup _{\Omega}(-u) \leq \sup _{\partial \Omega}(-u)+C(n, \lambda)|\Omega|^{\frac{1}{Q}}\|f\|_{L^{Q}(\Omega)} .
$$

It is worth to say before giving the proofs that, in general, whenever $F$ is homogeneous of degree 1 and not necessarily a concave/convex operator, our result is scale invariant.

Indeed, if $u$ is solution of $F\left(D_{\mathbb{H}^{n}}^{2 *} u(x), x\right)=f(x), r F(M, x)=F(r M, x), r>0$ and $F(0, x)=0$, then $v_{r}(x)=u\left(\delta_{r}(x)\right)$ satisfies

$$
\tilde{F}\left(D_{\mathbb{H}^{n}}^{2} v(x), x\right)=r^{2} f\left(\delta_{r}(x)\right),
$$

in $\delta_{r^{-1}}(\Omega)$, where

$$
\tilde{F}\left(D_{\mathbb{H}^{n}}^{2 *} v(x), x\right)=F\left(D_{\mathbb{H}^{n}}^{2 *} v(x), \delta_{r}(x)\right)=r^{2} F\left(D_{\mathbb{H}^{n}}^{2 *} u\left(\delta_{r}(x)\right), \delta_{r}(x)\right) .
$$

On the other hand, we apply to $v$ the previous result obtaining

$$
\sup _{\delta_{r^{-1}}(\Omega)} v \leq \sup _{\partial \delta_{r^{-1}}(\Omega)} v+C(n, \lambda)\left|\delta_{r^{-1}}(\Omega)\right|^{\frac{1}{Q}}\left\|r^{2} f\left(\delta_{r}(x)\right)\right\|_{L^{Q}\left(\delta_{r^{-1}}(\Omega)\right)} .
$$

Thus, $\sup _{\delta_{r^{-1}}(\Omega)} v=\sup _{\Omega} u, \sup _{\partial \delta_{r^{-1}}(\Omega)} v=\sup _{\partial \Omega} u$,

$$
\left|\delta_{r^{-1}}(\Omega)\right|^{\frac{1}{Q}}=r^{1-\frac{1}{Q}}|\Omega|^{\frac{1}{Q}},
$$

and

$$
\left\|r^{2} f\left(\delta_{r}(x)\right)\right\|_{L Q_{\left(\delta^{-1}(\Omega)\right)}}=r^{-1+\frac{1}{Q}}\|f\|_{L} Q_{(\Omega)} .
$$

Hence, substituting in (27), we get

$$
\sup _{\Omega} u \leq \sup _{\partial \Omega} u+C(n, \lambda)|\Omega|^{\frac{1}{Q}}\|f\|_{L^{Q}(\Omega)} .
$$

Proof of Corollary 4.1 Let $F$ be a uniformly horizontally degenerate fully nonlinear operators such that there exists a matrix with constant coefficients $B_{0} \in \mathcal{A}_{\lambda, \Lambda}$ such that in $\Omega$ :

$$
\operatorname{Tr}\left(B_{0} M\right) \geq F(M) .
$$


Let $u \in C^{2}(\Omega) \cap C(\bar{\Omega})$ be a solution of

$$
F\left(D^{2 *} u\right) \geq f(x), \Omega .
$$

Then, $\operatorname{Tr}\left(B_{0} D^{2 *} u(x)\right) \geq F\left(D^{2 *} u\right) \geq f(x)$. Let us denote by $v_{B_{0}}$ the solution of the problem

$$
\left\{\begin{array}{l}
\operatorname{Tr}\left(B_{0} D^{2 *} v_{B_{0}}(x)\right)=f(x), \quad \Omega \\
v_{B_{0}}=u, \quad \partial \Omega
\end{array}\right.
$$

Then, by the usual weak maximum principle results for the linear operator $\operatorname{Tr}\left(B_{0} D^{2 *}\right.$.), we get that $u \leq v_{B_{0}}$ in $\bar{\Omega}$. Thus, applying Theorem 1.1 to $v_{B_{0}}$, we get:

$$
\sup _{\partial \Omega} u \leq v_{B_{0}} \leq \sup _{\partial \Omega} v_{B_{0}}+C(n, \lambda)|\Omega|^{\frac{1}{Q}}\|f\|_{L^{Q}(\Omega)} .
$$

As a consequence,

$$
\sup _{\partial \Omega} u \leq \sup _{\partial \Omega} u+C(n, \lambda)|\Omega|^{\frac{1}{Q}}\|f\|_{L^{Q}(\Omega)} .
$$

Proof of Corollary 4.2 Let $F$ be a concave operator such that $F(0)=0$. Then, there exists a matrix in $B_{0} \in \mathcal{A}_{\lambda, \Lambda}$ such that

$$
F\left(D_{\mathbb{H}^{n}}^{2 *} u(x)\right) \leq \operatorname{Tr}\left(B_{0} D_{\mathbb{H}^{n}}^{2 *} u(x)\right)
$$

Thus, applying Corollary 4.1 to

$$
F\left(D_{\mathbb{H}^{n}}^{2 *} u(x)\right) \geq f(x), \Omega,
$$

we get

$$
\sup _{\Omega} u \leq \sup _{\partial \Omega} u+C(n, \lambda)|\Omega|^{\frac{1}{Q}}\|f\|_{L^{Q}(\Omega)},
$$

by proving the result for the concave operators.

Proof of Corollary 4.3 If $F$ is convex, then $G(M)=-F(-M)$ is still a horizontal degenerate uniformly elliptic fully nonlinear operator such that $G(0)=0$, that is, concave $G$. In particular, if $u \in C^{2}(\Omega) \cap C(\bar{\Omega})$ is solution of

$$
F\left(D_{\mathbb{H}^{n}}^{2 *} u(x)\right) \leq f
$$

in $\Omega$, where $f \in L^{Q}(\Omega)$, then

$$
G\left(D_{\mathbb{H}^{n}}^{2 *}(-u(x))\right) \geq-f(x)
$$

in $\Omega$, where $G$ is concave. Applying previous Corollary 4.2 for concave operators, we get

$$
\sup _{\Omega}(-u) \leq \sup _{\partial \Omega}(-u)+C(n, \lambda)|\Omega|^{\frac{1}{Q}}\|f\|_{L^{Q}(\Omega)}
$$

concluding the proof. 


\section{References}

1. Alvino, A., Trombetti, G.: Sulle migliori costanti di maggiorazione per una classe di equazioni ellittiche degeneri. Ricerche Mat. 27(2), 413-428 (1978)

2. Arcozzi, N., Ferrari, F.: Metric normal and distance function in the Heisenberg group. Math. Z. 256(3), 661-684 (2007)

3. Arcozzi, N., Ferrari, F.: The Hessian of the distance from a surface in the Heisenberg group. Annales Academia Scientiarum Fennica Mathematica 33, 35-63 (2008)

4. Arcozzi, N., Ferrari, F., Montefalcone F.: CC-distance and metric normal of smooth hypersurfaces in sub-Riemannian two-step Carnot groups. arXiv:0910.5648 (2009)

5. Bardi, M., Mannucci, P.: Comparison principles for equations for Monge-Ampère type in Carnot groups: a direct proof Geometric methods in PDE's, 41-51, Lect. Notes Semin. Interdiscip. Mat., 7, Semin. Interdiscip. Mat. (S.I.M.), Potenza, (2008)

6. Bardi, M., Mannucci, P.: Comparison principles for subelliptic equations of Monge-Ampère type. Boll. Unione Mat. Ital. (9) 1(2), 489-495 (2008)

7. Bonfiglioli, A., Lanconelli, E., Uguzzoni, F.: Stratified Lie groups and potential theory for their sub-Laplacians. Springer Monographs in Mathematics. Springer, Berlin (2007)

8. Bony, J.-M.: Principe du maximum et inégalité de Harnack pour les opérateurs elliptiques dégénérés. Séminaire de Théorie du Potentiel, dirigé par M. Brelot, G. Choquet et J. Deny:1967/1968, Exp. 1020 pp. Secrétariat mathématique, Paris (1969)

9. Bony, J.-M.: Principe du maximum, inégalite de Harnack et unicité du problème de Cauchy pour les opérateurs elliptiques dégénérés. Ann. Inst. Fourier (Grenoble) 19(1), 277-304 (1969)

10. Capogna, L., Danielli, D., Pauls, S.D., Tyson, J.T.: An introduction to the Heisenberg group and the sub-Riemannian isoperimetric problem. PM 259 Birkhäuser (2007)

11. Caffarelli, L., Cabré, X.: Fully nonlinear elliptic equations. American Mathematical Society Colloquium Publications, 43. American Mathematical Society, Providence, RI, pp. vi+104 (1995)

12. Danielli, D., Garofalo, N., Nhieu, D.M.: Notions of convexity in Carnot groups. Comm. Anal. Geom. 11(2), 263-341 (2003)

13. Danielli, D., Garofalo, N., Nhieu, D. M.: On the best possible character of the $L^{Q}$ norm in some a priori estimates for non-divergence form equations in Carnot groups. Proc. Am. Math. Soc. 131(11), 3487-3498 (electronic) (2003)

14. Ferrari, F.: Rearrangements and an obstacle problem for degenerate variational inequalities. Ricerche Mat. 47(2), 257-275 (1999)

15. Ferrari, F.: A Steiner formula in the Heisenberg group for Carnot-Charathéodory balls ( Subelliptic PDE's and applications to geometry and finance), 133-143, Lect. Notes Semin. Interdiscip. Mat., 6, Semin. Interdiscip. Mat. (S.I.M.), Potenza, (2007)

16. Franchi, B., Serapioni, R., Serra Cassano, F.: Rectifiability and perimeter in the Heisenberg group. Math. Ann. 321, 479-531 (2001)

17. Franchi, B., Serapioni, R., Serra Cassano, F.: On the structure of finite perimeter sets in step 2 Carnot groups. J. Geom. Anal. 13, 421-466 (2003)

18. Gilbarg, D., Trudinger, N.S.: Elliptic partial differential equations of second order. Reprint of the 1998 edition. Classics in Mathematics. Springer, Berlin, pp. xiv+517 (2001)

19. Gutierrez, C.E., Lanconelli, E.: Maximum principle, nonhomogeneous Harnack inequality, and Liouville theorems for X-elliptic operators. Comm. Partial Differ. Equ. 28, 1833-1862 (2003)

20. Gutierrez, C.E., Montanari, A.: Maximum and comparison principles for convex functions on the Heisenberg group. Comm. Partial Differ. Equ. 29(9-10), 1305-1334 (2004)

21. Pansu, P.: Une inégalité isopérimétrique sur le groupe de Heisenberg. C. R. Acad. Sci. Paris Sér. I Math. 295(2), 127-130 (1982)

22. Monti, R., Serra Cassano, F.: Surface measures in Carnot-Carathéodory spaces. Calc. Var. Partial Differ. Equ. 13, 339-376 (2001)

23. Talenti, G.: Elliptic equations and rearrangements. Ann. Scuola Norm. Sup. Pisa Cl. Sci. (4) 3(4), 697-718 (1976) 